\title{
On the BiHamiltonian Structure of the Supersymmetric KdV Hierarchies. A Lie Superalgebraic Approach
}

\author{
Carlo Morosi $^{1}$, Livio Pizzocchero ${ }^{2}$ \\ ${ }^{1}$ Dipartimento di Matematica, Politecnico di Milano, Piazza L. da Vinci 32, I-20133 Milano, \\ Italy \\ ${ }^{2}$ Dipartimento di Matematica, Università di Milano, Via C. Saldini 50, I-20133 Milano, Italy, \\ and Istituto Nazionale di Fisica Nucleare, Sezione di Milano, Italy
}

Received: July 10, 1992 / in revised form: April 12, 1993

\begin{abstract}
We give a Lie superalgebraic interpretation of the biHamiltonian structure of known supersymmetric KdV equations. We show that the loop algebra of a Lie superalgebra carries a natural Poisson pencil, and we subsequently deduce the biHamiltonian structure of the supersymmetric $\mathrm{KdV}$ hierarchies by applying to loop superalgebras an appropriate reduction technique. This construction can be regarded as a superextension of the Drinfeld-Sokolov method for building a KdVtype hierarchy from a simple Lie algebra.
\end{abstract}

\section{Introduction}

The possibility of superextensions of the integrable evolution equations has been considered in the literature in the last ten years. In particular, the construction of super Korteweg-de Vries hierarchies has been extensively discussed (an exhaustive bibliography can be found in [IK]).

Here, we are interested only in supersymmetric theories (from now on denoted by $\mathrm{sKdV}$ ), which are invariant under space supersymmetries; so, we will confine our attention to the hierarchies introduced in [MR, Mat, LM], and we will not consider the superequations proposed in [Kup, GO].

Recently, the biHamiltonian structures and the recursion operators for the known sKdV hierarchies have been determined. This remarkable result, due to [OP] and, independently, to [FMR], has been obtained by applying $R$-matrix theory to the algebra of super pseudo-differential operators in a suitable superderivation. In this framework, it is essential that each one of the proposed sKdV hierarchies admits a Lax formulation.

In this paper, we are discussing the biHamiltonian structure of sKdV hierarchies from a different point of view, which is based on the theory of simple Lie superalgebras. The differences and the existing relations between the $R$-matrix approach and the Lie algebraic approach are better understood if they are preliminarily discussed in the framework of classical integrable systems.

For definiteness, let us consider the ordinary KdV equation $q_{t}=-q_{x x x}+6 q q_{x}$, where the range of the space variable $x$ is assumed to be the one-dimensional 
torus $\mathbb{R} / \mathbb{Z}$ (or, alternatively, the real line $\mathbb{R}$ ). The associated Lax formulation, depending on the Lax operator

$$
L:=\partial_{x}^{2}-q
$$

can be interpreted as selecting an affine subspace in the algebra of pseudo-differential operators $\sum_{k<+\infty} u_{k}(x) \partial_{x}^{k}$, where $\partial_{x}:=\partial / \partial x$. This algebra possesses a natural $R$-matrix, namely the difference between the projections on the purely differential and the purely integrational parts. So, starting from the general biHamiltonian structure existing in any associative algebra endowed with an $R$-matrix [Sem], the biHamiltonian structure of the $\mathrm{KdV}$ hierarchy can be obtained by simply reducing the general structure to the Lax submanifold formed by differential operators of the form $-q(x)+\partial_{x}^{2}$. An analogous technique has been applied in [OP] for the construction of the biHamiltonian structure of the known sKdV hierarchies; this result rests on suitable supersymmetric extensions of the Lax operator and on the choice of an appropriate algebra of super pseudo-differential operators.

As it is known, the biHamiltonian structure of the classical $\mathrm{KdV}$ can also be obtained by a Lie algebraic approach. Indeed, let us consider the Lie algebra $g:=\operatorname{sl}(2)$, and the associated loop algebra $\mathscr{G}$, formed by the smooth functions $V: \mathbb{R} / \mathbb{Z} \rightarrow g$. We can regard $\mathscr{G}$ as a biHamiltonian manifold, endowed with the so-called Zakharov-Shabat biHamiltonian structure; this structure consists of a pair $P, Q$ of compatible Poisson tensors, whose expressions at any point $V \in \mathscr{G}$ are given by the operators

$$
P_{V}:=\partial_{x}+[V, \cdot], \quad Q_{V}:=[A, \cdot]
$$

acting on $\mathscr{G}$, where $A$ is a fixed element of $g$. In this framework, the $\mathrm{KdV}$ biHamiltonian structure is recovered by setting $A:=\left(\begin{array}{ll}0 & 0 \\ 1 & 0\end{array}\right)$ and by appropriately reducing $P$ and $Q$ to the submanifold $\left\{V \in \mathscr{G} \mid V=\left(\begin{array}{ll}0 & 1 \\ q & 0\end{array}\right)\right\}[\mathrm{CMP}]$.

The choice of $A$ and of the reduction submanifold can be interpreted from a Lie algebraic viewpoint. Its relation with the Lax formalism is illustrated by the remark that, for $\lambda \in \mathbb{C}$, the equation

$$
\Psi_{x}+V \Psi+\lambda A \Psi=0
$$

with $V$ in the reduction submanifold and $A$ as above, implies $\Psi=\left(\begin{array}{c}\psi \\ -\psi_{x}\end{array}\right)$, where $\psi$ is a solution of the eigenvalue problem $L \psi=\lambda \psi$ for the Lax operator (1.1). Also, Eq. (1.3) allows an interpretation of the Zakharov-Shabat biHamiltonian structure; in fact, if this equation is coupled with an evolution equation

$$
\Psi_{t}+U \Psi=0
$$

with $U \in \mathscr{G}$, the compatibility condition between (1.3) and (1.4) is

$$
V_{t}=P_{V} U+\lambda Q_{V} U
$$

Summarizing, for the KdV case both an operator and a Lie algebraic approach are possible. As it is known, both techniques can be extended to integrable systems associated to Lax problems of any order $n$ in $\dot{\partial}_{x}$, giving rise to the biHamiltonian structures for the Gel'fand-Dikii hierarchies. It may be worthwhile to remark that the Lie algebraic approach also works for different Lax problems, e.g. the one encompassing the AKNS hierarchy [New]. 
As for the Lie algebraic approach, a systematic treatment has been developed in [DS], allowing one to associate to any simple Lie algebra a biHamiltonian hierarchy of KdV-type. This result is fundamental, since it enlightens the deep connection between Lie algebras and integrable Hamiltonian systems and also allows a systematic construction and classification of the Lax pairs for KdV-type equations.

A central point in the Drinfeld-Sokolov theory is that, for any simple Lie algebra $g$, the associated loop algebra $\mathscr{G}$ can be viewed as an infinite-dimensional biHamiltonian manifold with the structure (1.2), depending on a distinguished element $A$ of $g$. If $A$ is chosen according to a well defined algebraic criterion, and a suitable reduction is applied, one obtains a KdV-type thoery; the reduction depends on the choice of a second distinguished element $B$ of $g$. A recent reformulation of this Lie algebraic approach, making transparent its geometrical meaning, has been developed in [CMP, CP].

Obviously enough, it is of great interest to extend the Drinfeld-Sokolov theory to the framework of supermathematics. Interesting results in this direction were obtained in [IK], where a Lie algebraic interpretation was given for the Lax operator of the so-called $N=1 \mathrm{sKdV}$ of Mathieu [Mat].

In this paper, we will discuss the superextension of the other relevant aspect of the Drinfeld-Sokolov theory, namely the biHamiltonian structure of loop algebras and its geometrical reduction; some results concerning Lax pairs, which are strictly connected with the content of the present paper, will be discussed elsewhere [MP].

We will work on loop algebras associated to simple Lie superalgebras and will equip them with a suitable superextension of the Zakharov-Shabat structure (1.2). We will adapt to this framework the Drinfeld-Sokolov criterion for choosing the distinguished elements $A$ and $B$ mentioned above, and we will apply to loop superalgebras the natural generalization of the reduction technique introduced in [CMP].

The paper is organized as follows, Section 2 contains an essential summary of definitions and properties of supermanifolds to be used in the rest of the paper, along with a review of some sKdV hierarchies, known in the literature, that will be reinterpreted from the superalgebraic viewpoint; this section is concluded by the statement of the reduction theorem for biHamiltonian manifolds to be applied in the subsequent sections. In Sect. 3 we discuss the superextension of the DrinfeldSokolov construction and the related criterion for choosing two distinguished elements $A, B$ in a Lie superalgebra. The reduction of the Zakharov-Shabat biHamiltonian structure is performed in Sect. 4,5 respectively for the Lie superalgebras $A(1,1)$ and osp $(2,2)$. In both cases, a sKdV hierarchy in two superfields is obtained as a natural outcome of the biHamiltonian reduction technique. As for $A(1,1)$, the hierarchy introduced in [MR] and the corresponding biHamiltonian structure constructed in [OP] are recovered. In the case of osp $(2,2)$, by applying the superextension of the Drinfeld-Sokolov criterion we get a two superfields theory, corresponding, up to a diffeomorphism, to the $N=2, \alpha=4$ superextension of $[\mathrm{LM}]$. A different choice of the distinguished elements in $\operatorname{osp}(2,2)$ is also considered in Sect. 5, so as to compare our criterion with alternative possibilities; in this way, another biHamiltonian structure is obtained, involving four superfields and giving rise by a subsequent reduction to the above mentioned $N=2$ theory. Section 6 is devoted to some comments on future developments, concerning in particular the relation between Lie superalgebras and Lax pairs. 


\section{Preliminaries about Supermanifolds and sKdV Equations. A biHamiltonian Reduction Theorem}

2.1. Some Supermathematics. We begin by considering a real or complex superalgebra, i.e. a $\mathbb{Z}_{2}$-graded algebra

$$
\Lambda=\Lambda_{0} \oplus \Lambda_{1}
$$

$\Lambda$ is assumed to be associative, with unit and commutative in the graded sense:

$$
a b=(-1)^{\operatorname{deg}(a) \operatorname{deg}(b)} b a
$$

for $a, b \in \Lambda_{0} \cup \Lambda_{1}$. The whole $\Lambda$, or its even part $\Lambda_{0}$ (which is a commutative algebra) will be the "set of scalars" on which to found all linear algebra, superanalysis and supergeometry. ${ }^{1}$ Our standard reference for linear algebra over $\Lambda$ is [Lei]. Among the existing approaches to superanalysis and supermanifolds, we have kept in mind, in particular, the setting proposed in [JP, VV].

In the sequel, we will be concerned with $\Lambda_{0}$-modules and/or $\Lambda$-supermodules [Lei]. If $\Sigma$ is a $\Lambda_{0}$-module, we will denote by $\Sigma^{*}$ the dual $\Lambda_{0}$-module, consisting of $\Lambda_{0}$-linear functionals from $\Sigma$ to $\Lambda_{0}$; similarly, if $E=E_{0} \oplus E_{1}$ is a $\Lambda$-supermodule, $E^{*}$ will be the dual supermodule, consisting of $\Lambda$-linear functionals from $E$ to $\Lambda$.

In the language of modules, a flat $(p, q)$-dimensional superspace $(p, q \in \mathbb{N})$ is defined to be a $\Lambda_{0}$-module which is isomorphic (within the category of $\Lambda_{0}$-modules) to the product module $\left(\Lambda_{0}\right)^{p} \times\left(\Lambda_{1}\right)^{q}$. If $E=E_{0} \oplus E_{1}$ is a free $\Lambda$-supermodule, with a basis formed by $p$ even and $q$ odd vectors, both its even part $E_{0}$ and its odd part $E_{1}$, when considered as $\Lambda_{0}$-modules, are flat superspaces, of dimensions $(p, q)$ and $(q, p)$ respectively. Flat superspaces are the starting point for superanalysis, and the local models for supermanifolds.

If $\mathscr{V}$ is a supermanifold, it is possible to define different types of tangent spaces at any point $v \in \mathscr{V}$ : the even tangent space $T_{v} \mathscr{V}$ and its odd complement $\overline{T_{v}} \mathscr{V}$, which are both $\Lambda_{0}$-modules; their direct sum $T_{v} \mathscr{V} \oplus \overline{T_{v}} \mathscr{V}$, which is a $\Lambda$ supermodule.

In this paper, the tangent space at $v$ will always mean the even one $T_{v} \mathscr{V}$ : for our purposes, it is not necessary to consider odd tangent vectors, so we exclude them from our general setting; the cotangent space at $v$, denoted by $T_{v}^{*} \mathscr{V}$, is defined to be the dual $\Lambda_{0}$-module. If $\mathscr{V}=\Sigma$, where $\Sigma$ is a flat superspace, we have two natural identifications $T_{v} \mathscr{V} \approx \Sigma, T_{v}^{*} \mathscr{V} \approx \Sigma^{*}$.

As far as we are concerned with even tangent vectors and covectors, the definition of Poisson tensor is the same as the classical one. More precisely, let $P$ be a (super) differentiable map which associates to any $v \in \mathscr{V}$ a skew-symmetric $\Lambda_{0}$-linear operator $P_{v}: T_{v}^{*} \mathscr{V} \rightarrow T_{v} \mathscr{V}$; for any pair $f, l$ of (super) differentiable $\Lambda_{0}$-valued functions on $\mathscr{V}$, let us define the bracket $\{f, l\}:=\langle d f, P d l\rangle$. We say that $P$ is a Poisson tensor if the associated bracket satisfies the Jacobi identity. The so-called "odd Poisson tensors," which would require a different definition [LMV], will not be considered here.

\footnotetext{
${ }^{1}$ In setting up superanalysis, it is customary to assume that $\Lambda$ carries a norm; since we are interested mainly in formal, i.e. algebraic aspects of superintegrable systems, we will not explicitly mention this fact in the sequel
} 
Two Poisson tensors $P$ and $Q$ on $\mathscr{V}$ are said to be compatible if the linear combination $P^{\lambda}:=P+\lambda Q$ is itself a Poisson tensor for each $\lambda \in \mathbb{R}$ (or $\mathbb{C}$ ) [Mag]. In this case, the one-parameter family $\left(P^{\lambda}\right)$ is said to be a Poisson pencil and $\mathscr{V}$ is said to be a biHamiltonian supermanifold.

Moreover, if $Q$ is invertible, we can define an (even) tensor $N=P \circ Q^{-1}$; it is straightforward to prove that $N$ has vanishing Nijenhuis torsion, provided that the latter be defined just as in the classical case [Nij]. As it is known in the classical framework, $N$ plays the role of a recursion operator, defining a hierarchy of biHamiltonian evolution equations. More precisely, if $K_{0}$ is a vector field preserving both $P$ and $Q$, a sequence $K_{n}(n=0,1,2, \ldots)$ of commuting vector fields, all of them preserving $P$ and $Q$, is defined recursively from $K_{0}$ via the relation $K_{n+1}=N K_{n}$. For a discussion of the Nijenhuis torsion in the framework of supermanifolds, see [LMV], where it is shown that this notion is not well defined in the odd case.

2.2. A Survey of the $\mathrm{sKdV}$ Theories. It is assumed that the above framework can be extended, at least in a formal sense, to infinite-dimensional supermanifolds (here and in the sequel, we will never aim to mathematical rigour in dealing with functional superspaces, which provide the local models for infinite-dimensional supermanifolds).

In the infinite-dimensional framework, it is natural to define a flat superspace to be a $\Lambda_{0}$-module which is isomorphic to a product $\mathscr{F}\left(I, \Lambda_{0}\right) \times \mathscr{F}\left(J, \Lambda_{1}\right)$, where $I, J$ are two sets and $\mathscr{F}\left(I, \Lambda_{0}\right), \mathscr{F}\left(J, \Lambda_{1}\right)$ are conveniently chosen $\Lambda_{0}$-modules of functions from $I$ to $\Lambda_{0}, J$ to $\Lambda_{1}$ respectively.

We now illustrate the (formal) biHamiltonian supermanifolds corresponding to some of the popular sKdV hierarchies; this will also be useful to standardize some notations.

Let us consider a real superalgebra $L=L_{0} \oplus L_{1}$, and set $\Lambda=L$ or, alternatively, define $\Lambda$ to be the complexification of $L$ (this means that $\Lambda_{k}=\left\{a+i b \mid a, b \in L_{k}\right\}$ for $\left.k=0,1\right)$. From now on, $K$ will denote the $(1,1)$-dimensional superspace $L_{0} \times L_{1}$, or the $(1,1)$ supercylinder $S_{0} \times L_{1}$, i.e. the product of the "even torus" $S_{0}:=L_{0} / \mathbb{Z} 1$ times the "odd $L_{1}$-line" $L_{1}$. A typical point of $K$ will be denoted by $(x, \theta)$.

The phase space for the $N=1 \mathrm{sKdV}$ hierarchy of Mathieu [Mat] is the infinitedimensional supermanifold $\mathscr{M}:=\mathscr{F}\left(K, \Lambda_{1}\right)$, whose points are odd superfields on $K$, i.e. (super) differentiable functions $\phi: K \rightarrow \Lambda_{1},(x, \theta) \mapsto \phi(x, \theta)$. The sKdV hierarchy of Manin and Radul [MR] lives in the supermanifold $\mathscr{M}=\mathscr{F}\left(K, \Lambda_{0}\right) \times \mathscr{F}\left(K, \Lambda_{1}\right)$, whose points are pairs $(a, \phi)$, where $a$ is an even and $\phi$ is an odd superfield on $K$. The same supermanifold can also be assumed as the phase space for another hierarchy, the so-called $N=2, \alpha=4 \mathrm{sKdV}$ of Mathieu and Laberge [LM]. (In the case $K=L_{0} \times L_{1}$, it is assumed that all superfields satisfy appropriate limiting conditions at infinity in the $x$ variable).

In dealing with the cotangent bundles of the supermanifolds corresponding to the hierarchies of Mathieu, Manin-Radul and Laberge-Mathieu, we assume that the dual module $\mathscr{F} *\left(K, \Lambda_{1}\right)$ is identified with $\mathscr{F}\left(K, \Lambda_{0}\right)$ via the $\Lambda_{0}$-bilinear map $\langle$, $\rangle$ given by

$$
\langle\delta \phi, \phi\rangle:=\int_{\boldsymbol{K}} d x d \theta \delta \phi(x, \theta) \phi(x, \theta)
$$


for each $\delta \phi \in \mathscr{F}\left(K, \Lambda_{0}\right), \phi \in \mathscr{F}\left(K, \Lambda_{1}\right)$, the integral being understood in the Berezin sense [Ber, VV, Lei, Ino]. Similarly, we assume $\mathscr{F} *\left(K, \Lambda_{0}\right) \approx \mathscr{F}\left(K, \Lambda_{1}\right)$; the dual module of $\mathscr{F}\left(K, \Lambda_{0}\right) \times \mathscr{F}\left(K, \Lambda_{1}\right)$ is identified with the set of pairs $(\delta a, \delta \phi) \in \mathscr{F}\left(K, \Lambda_{1}\right) \times \mathscr{F}\left(K, \Lambda_{0}\right)$, using the straighforward generalization of the bilinear form (2.3).

Hereafter, we have written down in Table 1 the Poisson pencils $(P+\lambda Q)$ for each of the three $\mathrm{sKdV}$ theories, together with the first vector fields $K_{0}, K_{1}, K_{2}, \ldots$ of the associated hierarchies. The explicit expressions given in the table contain the well known superderivative $D:=\theta \partial / \partial x+\partial / \partial \theta[\mathrm{SS}]$, fulfilling the identity $D^{2}=\partial_{x}$. Here and in the sequel, we frequently denote the $D$-derivatives with primes, and the $\partial_{x}$ derivatives with subscripts $x$, e.g. $a^{\prime \prime \prime}:=D^{3} a, \phi_{x x}:=\partial_{x}^{2} \phi$, etc.

\section{Table 1.}

\section{Mathieu theory}

Phase space: $\mathscr{M}=\mathscr{F}\left(K, \Lambda_{1}\right)$

Poisson pencil at a point $\phi \in \mathscr{M}$ :

$$
\begin{gathered}
P_{\phi}+\lambda Q_{\phi}: T_{\phi}^{*} \mathscr{M} \approx \mathscr{F}\left(K, \Lambda_{0}\right) \rightarrow T_{\phi} \mathscr{M} \approx \mathscr{F}\left(K, \Lambda_{1}\right) \\
\left(P_{\phi}+\lambda Q_{\phi}\right) \delta \phi=\left(-D^{5}+3 \phi D^{2}+\phi^{\prime} D+2 \phi_{x}\right) \delta \phi+\lambda\left(D^{2}\left(D^{3}-\phi\right)^{-1} D^{2}\right) \delta \phi
\end{gathered}
$$

First vector fields in the hierarchy:

$$
\begin{aligned}
& K_{0}(\phi)=\phi_{x} \\
& K_{1}(\phi)=\partial_{x}\left(-\phi_{x x}+3 \phi \phi^{\prime}\right) \\
& K_{2}(\phi)=\partial_{x}\left(\phi_{x x x x}-5 \phi_{x} \phi_{x}^{\prime}-5 \phi \phi_{x x}^{\prime}-5 \phi^{\prime} \phi_{x x}+10 \phi \phi^{\prime 2}\right)
\end{aligned}
$$

Manin-Radul theory

Phase space: $\mathscr{M}=\mathscr{F}\left(K, \Lambda_{0}\right) \times \mathscr{F}\left(K, \Lambda_{1}\right)$

Poisson pencil at a point $m=\left(\begin{array}{l}a \\ \phi\end{array}\right) \in \mathscr{M}$ :

$$
\begin{array}{r}
P_{m}+\lambda Q_{m}: T_{m}^{*} \mathscr{M} \approx \mathscr{F}\left(K, \Lambda_{1}\right) \times \mathscr{F}\left(K, \Lambda_{0}\right) \rightarrow T_{m} \mathscr{M} \approx \mathscr{F}\left(K, \Lambda_{0}\right) \times \mathscr{F}\left(K, \Lambda_{1}\right) \\
\left(P_{m}+\lambda Q_{m}\right)\left(\begin{array}{l}
\delta a \\
\delta \phi
\end{array}\right)=\left(\begin{array}{ll}
P_{a a} & P_{a \phi} \\
P_{\phi a} & P_{\phi \phi}
\end{array}\right)\left(\begin{array}{l}
\delta a \\
\delta \phi
\end{array}\right)+\lambda\left(\begin{array}{cc}
-D^{3}+\phi & -D^{2} \\
-D^{2}
\end{array}\right)\left(\begin{array}{l}
\delta a \\
\delta \phi
\end{array}\right)
\end{array}
$$

where the operators $P_{a a}, P_{a \phi}$, etc. are given by:

$$
\begin{aligned}
P_{a a}:= & D^{7}-3 \phi D^{4}+4 a D^{3}+\left(2 a^{\prime}-3 \phi_{x}\right) D^{2}+2 a_{x} D-3 a \phi+3 \phi \phi^{\prime}+a^{\prime \prime \prime}-\phi_{x x} \\
& -a^{\prime} D^{-1}\left(\phi^{\cdot}\right)-\phi D^{-1}(a D \cdot)-\phi D^{-1}\left(\phi D^{-1}(\phi \cdot)\right)-\phi D^{-1}\left(\phi_{x} \cdot\right)+\phi_{x} D^{-1}(\phi \cdot) \\
P_{a \phi}:= & D^{6}-2 \phi D^{3}+4 a D^{2}-\phi_{x} D+2 a_{x}+\phi D^{-1}(\phi D \cdot) \\
P_{\phi a}:= & D^{6}+2 \phi D^{3}+\left(4 a-2 \phi^{\prime}\right) D^{2}+\phi_{x} D+2 a_{x}-\phi^{\prime \prime \prime}+\phi^{\prime} D^{-1}(\phi \cdot) \\
P_{\phi \phi}:= & 4 \phi D^{2}+2 \phi_{x}
\end{aligned}
$$

First vector fields in the hierarchy:

$$
\begin{aligned}
& K_{0}(m)=\left(\begin{array}{l}
a_{x} \\
\phi_{x}
\end{array}\right) \\
& K_{1}(m)=\partial_{x}\left(\begin{array}{c}
-a_{x x}-3 a^{2}+3 \phi a^{\prime} \\
-\phi_{x x}+3 \phi \phi^{\prime}-6 a \phi
\end{array}\right)
\end{aligned}
$$


Table 1 (continued)

\section{Laberge-Mathieu theory}

Phase space: $\mathscr{M}=\mathscr{F}\left(K, \Lambda_{0}\right) \times \mathscr{F}\left(K, \Lambda_{1}\right)$

Poisson pencil at a point $m=\left(\begin{array}{l}a \\ \phi\end{array}\right) \in \mathscr{M}$ :

$$
\begin{gathered}
P_{m}+\lambda Q_{m}: T_{m}^{*} \mathscr{M} \approx \mathscr{F}\left(K, \Lambda_{1}\right) \times \mathscr{F}\left(K, \Lambda_{0}\right) \rightarrow T_{m} \mathscr{M} \approx \mathscr{F}\left(K, \Lambda_{0}\right) \times \mathscr{F}\left(K, \Lambda_{1}\right) \\
\left(P_{m}+\lambda Q_{m}\right)\left(\begin{array}{l}
\delta a \\
\delta \phi
\end{array}\right)=\left(\begin{array}{ll}
P_{a a} & P_{a \phi} \\
P_{\phi a} & P_{\phi \phi}
\end{array}\right)\left(\begin{array}{l}
\delta a \\
\delta \phi
\end{array}\right)+\lambda\left(\begin{array}{cc}
D^{2} \\
D^{2}
\end{array}\right)\left(\begin{array}{l}
\delta a \\
\delta \phi
\end{array}\right)
\end{gathered}
$$

where:

$$
\begin{array}{ll}
P_{a a}=D^{3}-\phi, & P_{a \phi}=-8 a D^{2}+4 a^{\prime} D-8 a_{x} \\
P_{\phi a}=-8 a D^{2}+4 a^{\prime} D-4 a_{x}, & P_{\phi \phi}=4 D^{5}-12 \phi D^{2}-4 \phi^{\prime} D-8 \phi_{x} .
\end{array}
$$

First vector fields in the hierarchy:

$$
\begin{aligned}
& K_{0}(m)=\left(\begin{array}{l}
a_{x} \\
\phi_{x}
\end{array}\right) \\
& K_{1}(m)=\partial_{x}\left(\begin{array}{c}
\phi^{\prime}-8 a^{2} \\
4 a^{\prime \prime \prime}-16 a \phi
\end{array}\right) \\
& K_{2}(m)=4 \partial_{x}\left(\begin{array}{c}
a_{x x}+16 a^{3}+3 \phi a^{\prime}-6 a \phi^{\prime} \\
\phi_{x x}-3 \phi \phi^{\prime}+48 a^{2} \phi-12 a^{\prime} a_{x}-24 a a^{\prime \prime \prime}
\end{array}\right)
\end{aligned}
$$

We wish to emphasize the following facts:

i) The formulation adopted here for the Leberge-Mathieu biHamiltonian structure seems to be different from the usual one [LM, OP], where the phase space is taken to be a space of $\Lambda_{0}$-valued functions $\Phi\left(x, \theta, \theta^{\prime}\right)$ of one commuting variable $x$ and two anticommuting variables $\theta, \theta^{\prime}$. Indeed, if we consider the $(1,2)$ superspace or supercylinder $K^{\prime}:=K \times L_{1}$, a (super) differentiable function $\Phi: K^{\prime} \rightarrow \Lambda_{0},\left(x, \theta, \theta^{\prime}\right) \mapsto \Phi\left(x, \theta, \theta^{\prime}\right)$ can be uniquely represented as

$$
\Phi\left(x, \theta, \theta^{\prime}\right)=-2 i a(x, \theta)-\phi(x, \theta) \theta^{\prime},
$$

where $a, \phi$ are respectively an even and an odd superfield on $K$. The Poisson pencil and the vector fields of the Laberge-Mathieu hierarchy, as written in [OP], acquire the expression given in Table 1 under the diffeomorphism $\mathscr{F}\left(K^{\prime}, \Lambda_{0}\right) \rightarrow \mathscr{F}\left(K, \Lambda_{0}\right) \times \mathscr{F}\left(K, \Lambda_{1}\right), \Phi \mapsto(a, \phi)$.

ii) The Mathieu sKdV theory can be viewed as a reduction of both the Manin-Radul and the Laberge-Mathieu theories to the submanifold $\mathscr{M}_{0}:=\left\{\left(\begin{array}{l}a \\ \phi\end{array}\right) \in \mathscr{M} \mid a=0\right\}$. Indeed, for the Manin-Radul theory the first vector field $K_{0}$ is tangent to $\mathscr{M}_{0}$, and this submanifold is invariant for the recursion operator $N=P \circ Q^{-1}$, which implies that $K_{1}, K_{2}, \ldots$ are also tangent to $\mathscr{M}_{0}$. In the Laberge-Mathieu case, the first vector field $K_{0}$ is again tangent to $\mathscr{M}_{0}$ and the submanifold is invariant for the squared operator $N^{2}$. So, the even order vector fields $K_{2}, K_{4}, \ldots$ are also tangent. In both cases, restriction of the tangent vector fields to the submanifold $\mathscr{M}_{0}$ gives the Mathieu hierarchy. 
Also, the biHamiltonian structure of the Mathieu theory can be viewed as a restriction of either the Manin-Radul or the Laberge-Mathieu structures. The details of the reduction for the Manin-Radul biHamiltonian structure can be found in [OP], who show that the Poisson tensors $P$ and $Q$ of Manin-Radul can be separately restricted to the submanifold $a=0$, giving rise to the Poisson tensors of the Mathieu theory (Oevel and Popowicz use the term "Dirac reduction" to indicate the restriction). More precisely, let us denote respectively with $Q^{[M]}, P^{[M]}$ and $Q^{[M R]}, P^{[M R]}$ the Poisson tensors of the hierarchies of Mathieu and Manin-Radul. If $m=\left(\begin{array}{l}0 \\ \phi\end{array}\right)$ is a point of the submanifold $\mathscr{M}_{0}$, and $\delta \phi \in \mathscr{F}\left(K, \Lambda_{0}\right)$, the equation

$$
\left(\begin{array}{l}
0 \\
\dot{\phi}
\end{array}\right)=P_{m}^{[M R]}\left(\begin{array}{l}
\delta a \\
\delta \phi
\end{array}\right)
$$

in the unknown superfields $\dot{\phi} \in \mathscr{F}\left(K, \Lambda_{1}\right)$ and $\delta a \in \mathscr{F}\left(K, \Lambda_{0}\right)$, has a unique solution, with $\dot{\phi}$ given by $\dot{\phi}=P_{\phi}^{[M]} \delta \phi$. Similarly, if we admit formal invertibility for the superdifferential operator $\left(D^{3}-\phi\right)$, the equation

$$
\left(\begin{array}{l}
0 \\
\dot{\phi}
\end{array}\right)=Q_{m}^{[M R]}\left(\begin{array}{l}
\delta a \\
\delta \phi
\end{array}\right)
$$

implies $\dot{\phi}=Q_{\phi}^{[M]} \delta \phi$. As regards the Poisson tensors $P^{[L M]}, Q^{[L M]}$ of the LabergeMathieu theory, only the first one $P^{[L M]}$ can be restricted as above. Nevertheless, in this case we can invoke the so-called restriction of the Poisson pencil; this amounts to considering the equation

$$
\left(\begin{array}{l}
0 \\
\dot{\phi}
\end{array}\right)=\left(P_{m}^{[M R]}+\lambda Q_{m}^{[L M]}\right)\left(\begin{array}{l}
\delta a \\
\delta \phi
\end{array}\right)
$$

which is uniquely solved with respect to $\dot{\phi}, \delta a$ for each $\lambda$, with $\dot{\phi}$ given by $\dot{\phi}=\left(-4 P_{\phi}^{[M]}-\lambda^{2} Q_{\phi}^{[M]}\right) \delta \phi$.

2.3. A BiHamiltonian Reduction Theorem. The results we are presenting in this paper depend on the following reduction theorem.

Proposition 2.1. Let $\mathscr{V}$ be a supermanifold, equipped with a Poisson pencil $\left(P^{\lambda}\right)=(P+\lambda Q)$. Define a distribution $\mathscr{D}$ on $\mathscr{V}$ setting

$$
\mathscr{D}_{v}:=P_{v}\left(\operatorname{ker} Q_{v}\right)
$$

for $v \in \mathscr{V}$, and choose a symplectic leaf $\mathscr{S}$ of the Poisson tensor $Q$. The following statements hold:

i) The distribution $\mathscr{E}$ on $\mathscr{S}$, defined by

$$
\mathscr{E}_{s}:=T_{s} \mathscr{S} \cap \mathscr{D}_{s}
$$

for each $s \in \mathscr{S}$, is integrable.

ii) Assume the quotient space $\mathscr{M}:=\mathscr{S} / \mathscr{E}$ to be a supermanifold, denote by $\pi: \mathscr{S} \rightarrow \mathscr{M}$ the natural projection and by $T \pi: T \mathscr{S} \rightarrow T \mathscr{M}$ the tangent map. Then $\mathscr{M}$ carries a Poisson pencil, again denoted with $\left(P^{\lambda}\right)=(P+\lambda Q)$, such that, for $m \in \mathscr{M}, \delta m \in$ $T_{m}^{*} \mathscr{M}$

$$
P_{m}^{\lambda} \delta m=\left(T_{s} \pi\right)\left(P_{s}^{\lambda} \delta v\right)
$$


where: $s$ is any point of $\mathscr{S}$ such that $\pi(s)=m$ and $\delta v \in T_{s}^{*} \mathscr{V}$ is any covector such that $\delta v \uparrow \mathscr{D}_{s}=0, \delta v \uparrow T_{s} \mathscr{S}=\delta m_{\circ} T_{s} \pi$ (these conditions about $\delta v$ imply that $P_{s}^{\lambda} \delta v \in T_{s} \mathscr{S}$, so that the right-hand side of (2.10) is well defined).

Proposition 2.1 is a restatement, at the level of supermanifolds, of a reduction theorem for ordinary biHamiltonian manifolds [CMP], based on a general Hamiltonian reduction theorem formulated in [MaR]. The proof given in [CMP] is also formally valid in our super framework, if $T \mathscr{V}$ and the Poisson tensors $P, Q$ are intended as in Subsect. 2.1 (i.e., if we exclude from our considerations odd vector fields, odd Poisson tensors, etc.).

As we will show in the sequel, if the manifold $\mathscr{V}$ of Prop. 2.1 is chosen to be the loop algebra of an appropriately chosen simple Lie superalgebra, with a superextension of the Zakharov-Shabat biHamiltonian structure, the application of the above reduction technique produces on the quotient space the biHamiltonian structure of known sKdV hierarchies.

\section{The BiHamiltonian Structure of Loop Superalgebras}

3.1. Lie and Loop Superalgebras. By a Lie superalgebra over $\Lambda$, we mean a $\Lambda$ supermodule $g=g_{0} \oplus g_{1}$ with elements $U, V, W \ldots$, equipped with a $\Lambda$-bilinear map $[]:, g \times g \rightarrow g$, which is consistent with the grading $(\operatorname{deg}([U, V])=$ $\operatorname{deg}(U)+\operatorname{deg}(V))$ and satisfies, in the graded sense, the requirement of skewsymmetry and the Jacobi identity. Let $g$ be a Lie superalgebra over $\Lambda$, and let us assume that:

i) as a $\Lambda$-supermodule, $g$ is free and finite-dimensional;

ii) $g$ admits a nondegenerate, even, graded symmetric, ad-invariant bilinear form, i.e. a nondegenerate $\Lambda$-bilinear map $\langle,\rangle_{g}: g \times g \rightarrow \Lambda$ such that

$$
\begin{aligned}
\operatorname{deg}\left(\langle U, V\rangle_{g}\right)= & \operatorname{deg}(U)+\operatorname{deg}(V),\langle U, V\rangle_{g}=(-1)^{\operatorname{deg}(U) \operatorname{deg}(V)}\langle V, U\rangle_{g}, \\
& \langle[U, V], W\rangle_{g}=\langle U,[V, W]\rangle_{g} .
\end{aligned}
$$

In the applications we will discuss, $g$ will be a Lie superalgebra of matrices with entries in $\Lambda$, with the superbracket $[U, V]:=U V-(-1)^{\operatorname{deg}(U) \operatorname{deg}(V)} V U$; the adinvariant bilinear form will be the Berezin supertrace of the matrix product.

Independently of this concrete realization, which will be illustrated in the sequel, we now associate to $g$ a conveniently defined loop superalgebra. To this purpose, let us consider the $(1,1)$-dimensional superspace or supercylinder $K$ defined in Subsect. 2.2, and let us introduce a space $\mathscr{G}=\mathscr{F}(K, g)$ of (super) differentiable functions from $K$ to $g$ (again denoted typically by $U, V, W \ldots$. . $\mathscr{G}$ is a Lie superalgebra over $\Lambda$; its even (resp. odd) part $\mathscr{G}_{0}$ (resp. $\mathscr{G}_{1}$ ) is formed by the functions on $K$ with values in $g_{0}$ (resp. $g_{1}$ ), and the superbracket is defined pointwisely, i.e.,

$$
[U, V](x, \theta):=[U(x, \theta), V(x, \theta)]
$$

for $U, V \in \mathscr{G},(x, \theta) \in K$. A nondegenerate, graded symmetric, ad-invariant bilinear form $\langle,\rangle_{\mathscr{G}}: \mathscr{G} \times \mathscr{G} \rightarrow \Lambda$ is defined by setting

$$
\langle U, V\rangle_{\mathscr{S}}:=\int_{K} d x d \theta\langle U(x, \theta), V(x, \theta)\rangle_{g}
$$


This bilinear form will be employed to identify the dual supermodule $\mathscr{G}^{*}$ with $\mathscr{G}$ itself. Since

$$
\operatorname{deg}\left(\langle U, V\rangle_{\mathscr{G}}\right)=\operatorname{deg}(U)+\operatorname{deg}(V)+1
$$

we can assume $\left(\mathscr{G}_{0}\right)^{*} \approx \mathscr{G}_{1}$ and $\left(\mathscr{G}_{1}\right)^{*} \approx \mathscr{G}_{0}$ (as $\Lambda_{0}$-modules).

Let us now consider the infinite-dimensional supermanifold $\mathscr{V}:=\mathscr{G}_{1}$. A typical element of $\mathscr{G}_{1}$ will be denoted by $V$; an element of $\mathscr{G}_{0}$ will be typically denoted by $\delta V$, when regarded as a linear functional on $\mathscr{G}_{1}$. A pair of compatible, even Poisson tensors $P, Q$ are defined on $\mathscr{V}$ by setting, for each $V \in \mathscr{V}$,

$$
\begin{gathered}
P_{V}: T_{V}^{* \mathscr{V}} \approx \mathscr{G}_{0} \rightarrow T_{V} \mathscr{V} \approx \mathscr{G}_{1}, \quad P_{V} \delta V:=D(\delta V)+[V, \delta V], \\
Q_{V}: T_{V}^{*} \mathscr{V} \approx \mathscr{G}_{0} \rightarrow T_{V} \mathscr{V} \approx \mathscr{G}_{1}, \quad Q_{V} \delta V:=[A, \delta V],
\end{gathered}
$$

where $A$ is a fixed element of $g_{1}$ (to be identified with a constant function on the supercylinder $K$ ) and $D:=\theta \partial_{x}+\partial_{\theta}$.

The Poisson tensors (3.5), (3.6) define a supersymmetric Zakharov-Shabat biHamiltonian structure, extending to the case of loop superalgebras the biHamiltonian structure (1.2). The biHamiltonian manifold $\mathscr{V}=\mathscr{G}_{1}$ can be taken as the basis for application of the biHamiltonian reduction technique described in Prop. 2.1. As we will show in the sequel, this reduction allows us to recover the biHamiltonian structures of the sKdV theories (and, hopefully, of the superextensions of other integrable equations).

The symplectic leaf $\mathscr{S}$ appearing in Prop. 2.1 can be specified by requiring that it passes through a fixed element $B$ of $g_{1}$ (again identified with a constant function on $K$ ). So the whole construction depends on the choice of $g$ and the distinguished elements $A, B$; any triplet $(g, A, B)$, where $g$ is a Lie superalgebra with the properties i), ii) assumed at the beginning of this subsection, and $A, B \in g_{1}$, will be called a Drinfeld-Sokolov triplet.

The choice of the Drinfeld-Sokolov triplets corresponding to the $\mathrm{sKdV}$ equations will be discussed in the next subsections; the requirement that the elements $A, B$ of $g$ be odd (which is necessary in this framework) will be essential in this discussion.

3.2. How to choose g. Grassmann Envelopes of Simple Lie Superalgebras. A standard technique to build a Lie superalgebra over $\Lambda$ (with $\Lambda$ complex) consists in taking the Grassmann envelope [Ber] over $\Lambda$ of a complex Lie superalgebra.

Let $\gamma=\gamma_{0} \oplus \gamma_{1}$ be a Lie superalgebra over $\mathbb{C}$, i.e. a complex $\mathbb{Z}_{2}$-graded vector space with a Lie superbracket. We recall that the Grassmann envelope of $\gamma$ over $\Lambda$ is the tensor product $g:=\Lambda \underset{\mathbb{C}}{\otimes} \gamma$. This is a free supermodule over $\Lambda,{ }^{2}$ and it is a Lie superalgebra over $\Lambda$ with the superbracket

$$
[a \otimes U, b \otimes V]:=(-1)^{\operatorname{deg}(U) \operatorname{deg}(b)}(a b) \otimes[U, V]
$$

for $a, b \in \Lambda, U, V \in \gamma$. Assume we are given a $\mathbb{C}$-bilinear, graded symmetric, adinvariant form $\langle,\rangle_{\gamma}: \gamma \times \gamma \rightarrow \mathbb{C}$ and let us further suppose that $\langle,\rangle_{\gamma}$ is consistent

\footnotetext{
2 An element $U \in g$ is even iff it can be written as a finite sum $U=\sum a_{i} \otimes U_{i}$, with $a_{i} \in \Lambda, U_{i} \in \gamma$ and $\operatorname{deg}\left(a_{i}\right)+\operatorname{deg}\left(U_{i}\right)=0$ for each $i$; similarly, odd elements are defined by the condition $\operatorname{deg}\left(a_{i}\right)+\operatorname{deg}\left(U_{i}\right)=1$
} 
with the grading, i.e. $\langle U, V\rangle_{\gamma}=0$ for $U \in \gamma_{0}, V \in \gamma_{1}$. We define its envelope $\langle,\rangle_{g}: g \times g \rightarrow \Lambda$ setting

$$
\langle a \otimes U, b \otimes V\rangle_{g}:=(-1)^{\operatorname{deg}(U) \operatorname{deg}(b)}(a b)\langle U, V\rangle_{\gamma} .
$$

This is a bilinear map with the properties listed in item ii) of Subsect. 3.1.

Let us consider, for example, the case $\gamma=g l(p, q)$. We recall that, for any pair $(p, q)$ of positive integers, the set $g l(p, q)$ of all $(p+q) \times(p+q)$ complex matrices is a Lie superalgebra with the superbracket given by

$$
[U, V]:=U V-(-1)^{\operatorname{deg}(U) \operatorname{deg}(V)} V U \text {; }
$$

a $(p+q) \times(p+q)$ matrix $U$ is declared to be even (resp. odd) if it can be written in block form as

$$
U=\left(\begin{array}{ll}
X & 0 \\
0 & Y
\end{array}\right) \quad\left(\text { resp. } U=\left(\begin{array}{ll}
0 & W \\
Z & 0
\end{array}\right)\right),
$$

where $X$ is a $p \times p$ block, $Y$ is $q \times q, W$ is $p \times q$ and $Z$ is $q \times p$. A graded symmetric, ad-invariant, consistent bilinear form on $\gamma=g l(p, q)$ is the bilinear map

$$
\langle U, V\rangle_{\gamma}:=\operatorname{Str}(U V)
$$

where Str is the Berezin supertrace, i.e.

$$
\operatorname{Str}(U):=\left\{\begin{array}{ll}
\operatorname{Tr}(X)-\operatorname{Tr}(Y), & \text { for } U \text { even } \\
0 & \text { for } U \text { odd }
\end{array} .\right.
$$

The Grassmann envelope of $g l(p, q)$ can be identified with the set $g l(p, q, \Lambda)$ of $(p+q) \times(p+q)$ matrices with entries in $\Lambda$. Any such matrix $U$ can be written in block form as

$$
U=\left(\begin{array}{ll}
X & W \\
Z & Y
\end{array}\right)
$$

with $X$ a $p \times p$ block, etc., and it is even (resp. odd) if and only if the blocks $X$ and $Y$ have even (resp. odd) entries, while $W$ and $Z$ have odd (resp. even) entries.

The Lie superbracket in $g l(p, q, \Lambda)$ can be expressed just as in (3.9), and the envelope of the bilinear form (3.11) is again the supertrace of the matrix product, where, for $U \in g l(p, q, \Lambda)$,

$$
\operatorname{Str}(U):=\left\{\begin{array}{ll}
\operatorname{Tr}(X)-\operatorname{Tr}(Y) & \text { if } U \text { is even } \\
\operatorname{Tr}(X)+\operatorname{Tr}(Y) & \text { if } U \text { is odd }
\end{array} .\right.
$$

In the applications presented in the rest of the paper, the Lie superalgebras $g$ over $\Lambda$ which we are going to employ are Grassmann envelopes of simple, complex Lie superalgebras; such simple superalgebras are either subalgebras $\gamma \subset g l(p, q)$ or quotients $\gamma=\hat{\gamma} / C$, where $\hat{\gamma}$ is a subalgebra of $g l(p, q)$ and $C$ is the center of $\hat{\gamma}$. Furthermore, they carry an ad-invariant form $\langle,\rangle_{\gamma}$ which is either the supertrace of the matrix product or the projection of this bilinear map on the quotient space $\hat{\gamma} / C$. Obviously enough, the Grassmann envelope of a subalgebra of $g l(p, q)$ can be identified with a subalgebra of $g l(p, q, \Lambda)$. 
In dealing with the loop superalgebra associated to a subalgebra of $g l(p, q, \Lambda)$, it is necessary to remember the standard rule for applying the superderivation $D$ to a matrix-valued function on $K$ [Cor]:

$$
D U:=\left(\begin{array}{rr}
D X & D W \\
-D Z & -D Y
\end{array}\right)
$$

if $U$ is represented as in (3.13).

\subsection{How to Choose A and B. Generators and Root Systems of Lie Superalgebras.} We assume here the standard terminology of the theory of Lie superalgebras over $\mathbb{C}$ (Cartan subalgebras, roots, etc.) [Kac, Cor]. We simply recall that, if $\gamma$ is a complex Lie superalgebra, any root $\alpha$ of $\gamma$ is said to be even (resp. odd) if it possesses a nonzero even (resp. odd) root vector.

Let now $r$ be a positive integer, $\tau$ a subset of $I:=\{1, \ldots, r\}$, and let $\mathscr{A}=\left(a_{i j}\right)$ be an $r \times r$ matrix (Cartan matrix), satisfying the $(m)$-condition stated in [Kac, 1977, p. 49]. Let $\hat{\gamma}$ be the contragradient Lie superalgebra over $\mathbb{C}$ corresponding to the pair $(\mathscr{A}, \tau) ; \hat{\gamma}$ possesses a set of generators $H_{i}, E_{i}, F_{i}(i \in I)$ such that

$$
\begin{gathered}
{\left[H_{i}, H_{j}\right]=0, \quad\left[H_{i}, E_{j}\right]=a_{i j} E_{j} \quad\left[H_{i}, F_{j}\right]=-a_{i j} F_{j}, \quad\left[E_{i}, F_{j}\right]=\delta_{i j} H_{j},} \\
\operatorname{deg} H_{i}=0 \quad \text { for } i \in I, \quad \operatorname{deg} E_{i}=\operatorname{deg} F_{i}=0 \quad \text { for } i \notin \tau, \\
\operatorname{deg} E_{i}=\operatorname{deg} F_{i}=1 \quad \text { for } i \in \tau .
\end{gathered}
$$

We assume that $\hat{\gamma}$ is finite-dimensional; in this case, it is simple, or simple modulo its center [Kac]. The linear span $\mathfrak{h}$ of $H_{1}, \ldots, H_{r}$ is a Cartan subalgebra of $\hat{\gamma}$, and any root $\alpha$ of $\gamma$ can be written as a linear combination $\alpha=\sum_{i \in I} m_{i} \alpha_{i}$, where $m_{1}, \ldots, m_{r}$ are integer coefficients and $\alpha_{1}, \ldots, \alpha_{r}$ are the simple roots, which satisfy

$$
\left\langle\alpha_{i}, H_{j}\right\rangle=a_{j i} \quad(i, j \in I) .
$$

If the simple roots are linearly independent, $\alpha$ is as above and $\alpha^{\prime}=\sum_{i \in I} m_{i}^{\prime} \alpha_{i}$ is another root, we say that $\alpha \leqq \alpha^{\prime}$ if $m_{i} \leqq m_{i}^{\prime}$ for each $i \in I$.

We now discuss a criterion for picking up in $\hat{\gamma}$ two distinguished elements $A, B$. It is natural to try to reproduce in this framework the Drinfeld-Sokolov scheme for building a $\mathrm{KdV}$ theory from a simple Lie algebra. As it is known, such a scheme suggests to take

$$
\begin{aligned}
& B:=\sum_{i} E_{i}, \\
& A:=\text { a root vector corresponding to the lowest root. }
\end{aligned}
$$

At the level of Lie superalgebras, there is the problem of combining the above presciptions with the basic requirement that $A$ and $B$ be odd. Obviously enough, the most natural way to obtain an odd $B$ consists in setting

$$
B:=\sum_{i \in \tau} E_{i}
$$

As for $A$, one is faced with the problem that the lowest root could fail to be odd (see, for example, the application discussed in Sect. 5). For dealing with this situation, 
we propose an adaptation of the Drinfeld-Sokolov prescription, which consists in setting

$$
A:=\text { a root vector corresponding to a minimal odd root }
$$

(i.e., an odd root $\alpha$ such that, for every odd root $\alpha^{\prime}, \alpha^{\prime} \leqq \alpha$ implies $\alpha^{\prime}=\alpha$ ). If the simple roots are not linearly independent, we take $A$ to be a root vector of a conveniently defined (odd) lowest root (see Sect. 4).

Once the distinguished pair $(A, B)$ has been chosen in $\hat{\gamma}$, the construction of a Drinfeld-Sokolov triplet is straightforward. First of all, we factor out from $\hat{\gamma}$ its center $C$, which may be nonzero (see Sect. 4); the quotient Lie superalgebra $\gamma:=\hat{\gamma} / C$ is simple, and possesses a nondegenerate ad-invariant form, unique up to a factor [Kac]. We consider in $\gamma$ the pair of distinguished elements $A$ (mod. $C$ ), $B$ (mod. $C$ ), again denoted by $A$ and $B$ for simplicity. Finally, we take the Grassmann envelope $g:=\Lambda \otimes \gamma$, and we pick up in $g$ the distinguished elements $1 \otimes A$, $1 \otimes B$, once again denoted by $A$ and $B$. The Drinfeld-Sokolov triplet $(g, A, B)$ defined in this way can be taken as a starting point for the application of the biHamiltonian reduction scheme of Prop. 2.1; applications of this procedure will be illustrated in the next sections.

\section{BiHamiltonian Reduction on $A(1,1)$}

Let us consider the Lie superalgebra

$$
\operatorname{sl}(2,2):=\{V \in \operatorname{gl}(2,2) \mid \operatorname{Str}(V)=0\} .
$$

This superalgebra can be identified with the contragradient Lie superalgebra associated to the Cartan matrix $\mathscr{A}:=\left(\begin{array}{rrr}0 & 1 & 0 \\ -1 & 0 & 1 \\ 0 & -1 & 0\end{array}\right)$ and the index set $\tau:=\{1,2,3\}$, the generators $H_{i}, E_{i}, F_{i}$ being given by

$$
\begin{aligned}
& H_{1}:=\left(\begin{array}{llll}
1 & 0 & 0 & 0 \\
0 & 0 & 0 & 0 \\
0 & 0 & 1 & 0 \\
0 & 0 & 0 & 0
\end{array}\right) \text {, } \\
& H_{2}:=\left(\begin{array}{cccc}
0 & 0 & 0 & 0 \\
0 & 1 & 0 & 0 \\
0 & 0 & 1 & 0 \\
0 & 0 & 0 & 0
\end{array}\right), \\
& H_{3}:=\left(\begin{array}{llll}
0 & 0 & 0 & 0 \\
0 & 1 & 0 & 0 \\
0 & 0 & 0 & 0 \\
0 & 0 & 0 & 1
\end{array}\right), \\
& E_{1}:=\left(\begin{array}{cccc}
0 & 0 & 1 & 0 \\
0 & 0 & 0 & 0 \\
0 & 0 & 0 & 0 \\
0 & 0 & 0 & 0
\end{array}\right), \\
& E_{2}:=\left(\begin{array}{rrrr}
0 & 0 & 0 & 0 \\
0 & 0 & 0 & 0 \\
0 & -1 & 0 & 0 \\
0 & 0 & 0 & 0
\end{array}\right) \text {, } \\
& E_{3}:=\left(\begin{array}{cccc}
0 & 0 & 0 & 0 \\
0 & 0 & 0 & 1 \\
0 & 0 & 0 & 0 \\
0 & 0 & 0 & 0
\end{array}\right) \text {, } \\
& F_{1}:=\left(\begin{array}{cccc}
0 & 0 & 0 & 0 \\
0 & 0 & 0 & 0 \\
1 & 0 & 0 & 0 \\
0 & 0 & 0 & 0
\end{array}\right), \\
& F_{2}:=\left(\begin{array}{rrrr}
0 & 0 & 0 & 0 \\
0 & 0 & -1 & 0 \\
0 & 0 & 0 & 0 \\
0 & 0 & 0 & 0
\end{array}\right) \\
& F_{3}:=\left(\begin{array}{llll}
0 & 0 & 0 & 0 \\
0 & 0 & 0 & 0 \\
0 & 0 & 0 & 0 \\
0 & 1 & 0 & 0
\end{array}\right) \text {. }
\end{aligned}
$$


The Dynkin diagram corresponding to the pair $(\mathscr{A}, \tau)$ is $\otimes-\otimes-\otimes$. The linear span $\mathfrak{h}$ of $H_{1}, H_{2}, H_{3}$ consists of the diagonal matrices with zero supertrace. The relevant informations about the set of nonzero roots of $\operatorname{sl}(2,2)$ are contained in the diagram ${ }^{3}$

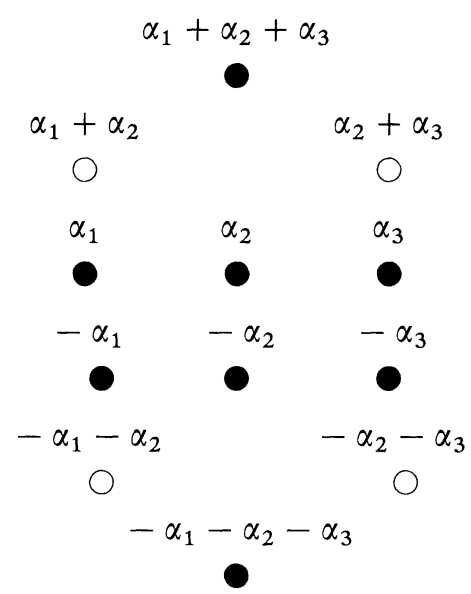

where:

i) $\alpha_{1}, \alpha_{2}, \alpha_{3}$ denote the simple roots, fulfilling (3.17);

ii) even roots are denoted by white circles $\bigcirc$ and odd roots by black circles

A root vector for the lowest root $-\alpha_{1}-\alpha_{2}-\alpha_{3}$ is the odd matrix

$$
A:=\left(\begin{array}{llll}
0 & 0 & 0 & 0 \\
0 & 0 & 0 & 0 \\
0 & 0 & 0 & 0 \\
1 & 0 & 0 & 0
\end{array}\right) .
$$

If we define $B$ to be the sum of the generators $E_{i}$, we find

$$
B:=\left(\begin{array}{rrrr}
0 & 0 & 1 & 0 \\
0 & 0 & 0 & 1 \\
0 & -1 & 0 & 0 \\
0 & 0 & 0 & 0
\end{array}\right) .
$$

The center of $\operatorname{sl}(2,2)$ consists of the scalar multiples of the identity matrix $I_{4}$. The quotient Lie superalgebra $\operatorname{sl}(2,2) / \mathbb{C} I_{4}$ is usually denoted by $A(1,1)$. The bilinear form $U, V \mapsto \operatorname{Str}(U V)$ is degenerate on $\operatorname{sl}(2,2)$, but it projects onto a nondegenerate

3 This diagram gives a redundant representation of the root system, due to the fact that the simple roots are not linearly independent: $\alpha_{3}=-\alpha_{1}$. This is an exceptional feature of the $s l(r, r)$ series [Cor], requiring an ad hoc definition of the lowest root. Following [FSS], we take the odd root $-\alpha_{1}-\alpha_{2}-\alpha_{3}=-\alpha_{2}$ as the lowest root 
bilinear form on $A(1,1)$. The Grassmann envelopes $\Lambda \underset{\mathbb{C}}{\otimes} \operatorname{sl}(2,2)$, and $\Lambda \underset{\mathbb{C}}{\otimes} A(1,1)$ can be identified with

$$
\operatorname{sl}(2,2, \Lambda):=\{V \in \operatorname{gl}(2,2, \Lambda) \mid \operatorname{Str}(V)=0\}, \quad A(1,1, \Lambda):=\operatorname{sl}(2,2, \Lambda) / \Lambda I_{4} .
$$

Computations in $A(1,1, \Lambda)$ are greatly simplified if we pick up a canonical representative $V$ in each equivalence class mod. $\Lambda I_{4}$. A simple rule for defining the canonical representative is the following: $V$ is the unique matrix in the equivalence class such that $V_{44}=0$ (equivalently, any other diagonal entry of $V$ could be set equal to zero). In this way we obtain an identification

$$
A(1,1, \Lambda) \approx\left\{V \in \operatorname{gl}(2,2, \Lambda) \mid \operatorname{Str}(V)=0, V_{44}=0\right\}
$$

and, under this identification, the superbracket in $A(1,1, \Lambda)$ is given by

$$
[U, V]:=U V-(-1)^{\operatorname{deg}(U) \operatorname{deg}(V)} U V+a I_{4}
$$

with $a \in \Lambda$ uniquely fixed by the condition $[U, V]_{44}=0 .{ }^{4}$ In the representation (4.7), which will be used from now on, the even and odd parts of $A(1,1, \Lambda)$ are given by

$$
\begin{gathered}
A(1,1, \Lambda)_{0}=\left\{U \in g l(2,2, \Lambda) \mid U=\left(\begin{array}{cccc}
u & v & \alpha & \beta \\
w & z & \gamma & \pi \\
\varepsilon & \rho & u+z & f \\
\xi & \eta & d & 0
\end{array}\right)\right\}, \\
A(1,1, \Lambda)_{1}=\left\{V \in g l(2,2, \Lambda) \mid V=\left(\begin{array}{cccc}
\rho & \mu & p & q \\
\sigma & \psi & r & s \\
h & k & -\rho-\psi & \delta \\
l & m & \chi & 0
\end{array}\right)\right\} .
\end{gathered}
$$

where Latin letters $h, k, l, \ldots$ denote even elements of $\Lambda$, while Greek letters $\alpha, \beta, \ldots$ stand for odd elements of $\Lambda$.

We now consider the loop superalgebra $\mathscr{G}=\mathscr{F}(K, A(1,1, \Lambda))$, and we apply the biHamiltonian reduction theorem to the supermanifold $\mathscr{G}_{1}$, equipped with the Poisson pencil $P+\lambda Q$ defined by (3.5), (3.6), and with $A$ as in (4.4).

We omit most of the computations, which are lengthy but straightforward. The symplectic leaf of $Q$ passing through $B$ (with $B$ as in (4.5)) is the submanifold

$$
\mathscr{S}=\left\{S \in \mathscr{G}_{1} \mid S=\left(\begin{array}{rrrr}
0 & 0 & 1 & 0 \\
\sigma & \psi & 0 & 1 \\
l & -1 & -\psi & 0 \\
h & k & \chi & 0
\end{array}\right)\right\}
$$

\footnotetext{
${ }^{4}$ We recall that $a I_{4}=\operatorname{diag}(a, a,-a,-a)$ if $a$ is odd
} 
with $h, k, l \in \mathscr{F}\left(K, \Lambda_{0}\right)$ and $\sigma, \psi, \chi \in \mathscr{F}\left(K, \Lambda_{1}\right)$. We have to consider the distributions $\mathscr{D}=P(\operatorname{ker} Q)$ and $\mathscr{E}=\mathscr{D} \cap T \mathscr{S}$. For $S$ as in (4.11), we have

$$
\mathscr{E}_{S}:=\left\{\dot{S} \in T_{S} \mathscr{G}_{1} \approx \mathscr{G}_{1} \mid \dot{S}=\left(\begin{array}{cccc}
0 & 0 & 0 & 0 \\
\dot{\sigma} & \dot{\psi} & 0 & 0 \\
\dot{l} & 0 & -\dot{\psi} & 0 \\
\dot{h} & \dot{k} & \dot{\chi} & 0
\end{array}\right)\right\}
$$

where the matrix elements $\dot{\sigma}, \dot{\psi}, \ldots$ are parametrized by four arbitrary superfields $d \in \mathscr{F}\left(K, \Lambda_{0}\right), \gamma, \xi, \eta \in \mathscr{F}\left(K, \Lambda_{1}\right)$ :

$$
\begin{aligned}
& \dot{\sigma}=\psi d-\gamma l+\xi+d^{\prime}+\psi \gamma^{\prime}+\gamma_{x}, \\
& \dot{\psi}=\gamma, \quad \dot{\chi}=\gamma k+d \psi-\xi-d^{\prime}, \\
& \dot{h}=k d+\chi \eta-d l-\eta \sigma+k \gamma^{\prime}-\xi^{\prime}, \\
& \dot{k}=d-\eta \psi-\eta^{\prime}, \quad \dot{l}=-d+\eta \psi-\eta^{\prime}-\gamma^{\prime} .
\end{aligned}
$$

Integration of the distribution $\mathscr{E}$ leads to the result that the quotient space $\mathscr{M}=\mathscr{S} / \mathscr{E}$ can be identified with $\mathscr{F}\left(K, \Lambda_{0}\right) \times \mathscr{F}\left(K, \Lambda_{1}\right)$. Modulo this identification, the canonical projection is $\pi: \mathscr{S} \rightarrow \mathscr{M}, S \mapsto \pi(S)=\left(\begin{array}{l}a \\ \phi\end{array}\right)$, where

$$
\begin{aligned}
a= & h-\frac{1}{4} k^{2}+\frac{1}{2} k l-\frac{1}{4} l^{2}-\frac{1}{2}(k-l) \psi^{\prime}-\frac{1}{4}\left(\psi^{\prime}\right)^{2}-\frac{1}{2}\left(k_{x}-l_{x}\right)-\frac{1}{2} \psi^{\prime \prime \prime}+l^{\prime} \psi+\sigma^{\prime} \\
& +\frac{1}{2}\left(-\psi_{x}+\sigma+\chi-k \psi+l \psi\right)\left(D^{-1}(k+l)+\psi\right) \\
\phi= & -\psi_{x}+\sigma+\chi-k \psi+l \psi
\end{aligned}
$$

A section of the bundle $\mathscr{S} \stackrel{\pi}{\rightarrow} \mathscr{M}$, which can be employed for computing the reduced Poisson pencil on $\mathscr{M}$, is the map

$$
\mathscr{M} \rightarrow \mathscr{S}, \quad m=\left(\begin{array}{l}
a \\
\phi
\end{array}\right) \mapsto S_{m}=\left(\begin{array}{rrrr}
0 & 0 & 1 & 0 \\
0 & 0 & 0 & 1 \\
0 & -1 & 0 & 0 \\
a & 0 & \phi & 0
\end{array}\right) .
$$

The final result is that the reduced Poisson pencil on the quotient space $\mathscr{M}$ is just the Poisson pencil of the Manin-Radul sKdV theory, given in Table 1.

For completeness, we remark that the same result is obtained if one applies the biHamiltonian reduction to the (non-simple) Lie superalgebra $g l(2,2, \Lambda)$, with $A$ and $B$ given again by (4.4) and (4.5).

\section{BiHamiltonian Reduction on $\operatorname{osp}(2,2)$}

Let us denote by osp $(2,2)$ the orthogonal-symplectic Lie superalgebra defined as in [Kac, 1977, p. 30]. This is a subalgebra of $g l(2,2)$; it is simple and can be identified with the contragradient Lie superalgebra associated to the Cartan matrix 
$\mathscr{A}:=\left(\begin{array}{ll}0 & 2 \\ 2 & 0\end{array}\right)$ and the set $\tau:=\{1,2\}$. The generators are [IK]:

$$
\begin{aligned}
& H_{1}:=\left(\begin{array}{rrrr}
1 & 0 & 0 & 0 \\
0 & -1 & 0 & 0 \\
0 & 0 & 1 & 0 \\
0 & 0 & 0 & -1
\end{array}\right), \quad H_{2}:=\left(\begin{array}{rrrr}
-1 & 0 & 0 & 0 \\
0 & 1 & 0 & 0 \\
0 & 0 & 1 & 0 \\
0 & 0 & 0 & -1
\end{array}\right) \text {, } \\
& E_{1}:=\left(\begin{array}{cccc}
0 & 0 & 0 & 0 \\
0 & 0 & 0 & 1 \\
1 & 0 & 0 & 0 \\
0 & 0 & 0 & 0
\end{array}\right), \quad E_{2}:=\left(\begin{array}{cccc}
0 & 0 & 0 & 1 \\
0 & 0 & 0 & 0 \\
0 & 1 & 0 & 0 \\
0 & 0 & 0 & 0
\end{array}\right) \text {, } \\
& F_{1}:=\left(\begin{array}{rrrr}
0 & 0 & 1 & 0 \\
0 & 0 & 0 & 0 \\
0 & 0 & 0 & 0 \\
0 & -1 & 0 & 0
\end{array}\right), \quad F_{2}:=\left(\begin{array}{rrrr}
0 & 0 & 0 & 0 \\
0 & 0 & 1 & 0 \\
0 & 0 & 0 & 0 \\
-1 & 0 & 0 & 0
\end{array}\right) .
\end{aligned}
$$

The Dynkin diagram corresponding to $(\mathscr{A}, \tau)$ is $\otimes-\otimes$, and the set of nonzero roots can be represented as

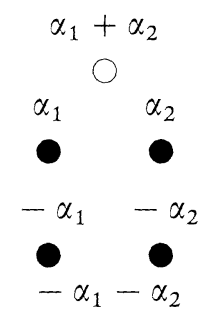

in terms of the simple roots $\alpha_{1}, \alpha_{2}$. In contrast with the case of Sect. 4 , the lowest root $-\alpha_{1}-\alpha_{2}$ is now even; so, we choose in substitution a minimal odd root, according to the adaptation of the Drinfeld-Sokolov criterion proposed in Subsect. 3.3. In the sequel, we consider in detail the choice $-\alpha_{2}$, corresponding to the root vector

$$
A=\left(\begin{array}{rrrr}
0 & 0 & 0 & 0 \\
0 & 0 & -1 & 0 \\
0 & 0 & 0 & 0 \\
1 & 0 & 0 & 0
\end{array}\right)
$$

(the choice of the minimal root $-\alpha_{1}$ gives a similar result). Following the prescriptions of Subsect. 3.3, we put

$$
B=E_{1}+E_{2}=\left(\begin{array}{cccc}
0 & 0 & 0 & 1 \\
0 & 0 & 0 & 1 \\
1 & 1 & 0 & 0 \\
0 & 0 & 0 & 0
\end{array}\right)
$$


The nondegenerate, ad-invariant bilinear form on $\operatorname{osp}(2,2)$ required for our construction is provided by the supertrace of the matrix product.

We now consider the Grassmann envelope $\operatorname{osp}(2,2, \Lambda):=\Lambda \underset{\mathbb{C}}{\otimes} \operatorname{osp}(2,2)$. A generic even element in $g l(2,2, \Lambda)$, can be written as

$$
U=\left(\begin{array}{rrrr}
p & 0 & \mu & v \\
0 & -p & \rho & -\sigma \\
\sigma & -v & q & r \\
\rho & \mu & s & -q
\end{array}\right), \quad V=\left(\begin{array}{rrrr}
\alpha & 0 & b & c \\
0 & -\alpha & d & f \\
f & c & \beta & \gamma \\
-d & -b & \varepsilon & -\beta
\end{array}\right),
$$

where, as usually, elements of $\Lambda_{0}$ (resp. $\Lambda_{1}$ ) are denoted by Latin (resp. Greek) letters. We now introduce the loop algebra $\mathscr{G}=\mathscr{F}(K, \operatorname{osp}(2,2, \Lambda))$, and we apply Prop. 2.1 to its odd part $\mathscr{G}_{1}$, viewed as a biHamiltonian supermanifold with the Poisson pencil $P+\lambda Q$ defined by (3.5), (3.6), $A$ being as in (5.3). The symplectic leaf $\mathscr{S}$ of the Poisson tensor $Q$ passing through $B$ is

$$
\mathscr{S}=\left\{S \in \mathscr{G}_{1} \mid S=\left(\begin{array}{rrrr}
\alpha & 0 & 0 & 1 \\
0 & -\alpha & d & f \\
f & 1 & \alpha & 0 \\
-d & 0 & \varepsilon & -\alpha
\end{array}\right)\right\}
$$

with $d, f \in \mathscr{F}\left(K, \Lambda_{0}\right)$ and $\alpha, \varepsilon \in \mathscr{F}\left(K, \Lambda_{1}\right)$. At any point $S$ of the symplectic leaf, the distribution $\mathscr{E}_{S}=P_{S}\left(\operatorname{ker} Q_{S}\right) \cap T_{S} \mathscr{S}$ is parametrized by two superfields $p \in$ $\mathscr{F}\left(K, \Lambda_{0}\right), \rho \in \mathscr{F}\left(K, \Lambda_{1}\right)$, and it is given by

$$
\mathscr{E}_{S}:=\left\{\dot{S} \in T_{S} \mathscr{G}_{1} \approx \mathscr{G}_{1} \mid \dot{S}=\left(\begin{array}{cccc}
\rho+p^{\prime} & 0 & 0 & 0 \\
0 & -\rho-p^{\prime} & \rho^{\prime} & 2 p f \\
2 p f & 0 & \rho+p^{\prime} & 0 \\
-\rho^{\prime} & 0 & -2 p \varepsilon & -\rho-p^{\prime}
\end{array}\right)\right\}
$$

with $f, \varepsilon$ as in (5.6). The quotient manifold $\mathscr{M}:=\mathscr{S} / \mathscr{E}$ can be identified with $\mathscr{F}\left(K, \Lambda_{0}\right) \times \mathscr{F}\left(K, \Lambda_{1}\right)$, and the natural projection is $\pi: \mathscr{S} \rightarrow \mathscr{M}, S \mapsto \pi(S)=\left(\begin{array}{c}a \\ \phi\end{array}\right)$, where

$$
a=\frac{1}{4} \frac{f_{x}}{f}-\frac{1}{2} \alpha^{\prime}+\frac{1}{2} d, \quad \phi=-2 a^{\prime}-2 \varepsilon f .
$$

A section of the bundle $\mathscr{S} \stackrel{\pi}{\rightarrow} \mathscr{M}$ is the map

$$
m=\left(\begin{array}{l}
a \\
\phi
\end{array}\right) \mapsto S_{m}=\left(\begin{array}{rccc}
0 & 0 & 0 & 1 \\
0 & 0 & 2 a & 1 \\
1 & 1 & 0 & 0 \\
-2 a & 0 & -a^{\prime}-\frac{1}{2} \phi & 0
\end{array}\right) .
$$

The reduction of the Poisson tensors $P, Q$ gives rise to the biHamiltonian structure of the Laberge-Mathieu theory (see Table 1). 
It may be of interest to consider the biHamiltonian reduction of the Poisson pencil corresponding to an alternative choice of $A$, namely

$$
A=\left(\begin{array}{rrrr}
0 & 0 & 1 & 0 \\
0 & 0 & -1 & 0 \\
0 & 0 & 0 & 0 \\
1 & -1 & 0 & 0
\end{array}\right),
$$

the matrix $B$ being the same as in (5.4). The new pair $(A, B)$ is known to be related to the Lax formulation for the $N=1$ Mathieu theory [IK]. If we apply the biHamiltonian reduction technique to $\operatorname{osp}(2,2, \Lambda)$ with this choice of $A$ and $B$, we find that the symplectic leaf $\mathscr{S}$ is parametrized by six superfields, and the quotient manifold $\mathscr{M}=\mathscr{S} / \mathscr{E}$ by four superfields $v, a, \sigma, \phi$, with $v, a \in \mathscr{F}\left(K, \Lambda_{0}\right)$ and $\sigma, \phi \in \mathscr{F}\left(K, \Lambda_{1}\right)$. The reduced Poisson pencil on $\mathscr{M}$ is given in Table 2.

Table 2. "Four superfields" theory

$$
\begin{aligned}
& \text { Poisson pencil at a point } m=\left(\begin{array}{l}
v \\
\sigma \\
a \\
\phi
\end{array}\right) \in \mathscr{M} \text { : } \\
& P_{m}+\lambda Q_{m}: T_{\phi}^{*} \mathscr{M} \approx \mathscr{F}\left(K, \Lambda_{1}\right) \times \mathscr{F}\left(K, \Lambda_{0}\right) \times \mathscr{F}\left(K, \Lambda_{1}\right) \times \mathscr{F}\left(K, \Lambda_{0}\right) \\
& \rightarrow T_{m} \mathscr{M} \approx \mathscr{F}\left(K, \Lambda_{0}\right) \times \mathscr{F}\left(K, \Lambda_{1}\right) \times \mathscr{F}\left(K, \Lambda_{0}\right) \times \mathscr{F}\left(K, \Lambda_{1}\right) \\
& \left(P_{m}+\lambda Q_{m}\right)\left(\begin{array}{c}
\delta v \\
\delta \sigma \\
\delta a \\
\delta \phi
\end{array}\right):=\left(\begin{array}{llll}
P_{v v} & P_{v \sigma} & P_{v a} & P_{v \phi} \\
P_{\sigma v} & P_{\sigma \sigma} & P_{\sigma a} & P_{\sigma \phi} \\
P_{a v} & P_{a \sigma} & P_{a a} & P_{a \phi} \\
P_{\phi v} & P_{\phi \sigma} & P_{\phi a} & P_{\phi \phi}
\end{array}\right)\left(\begin{array}{c}
\delta v \\
\delta \sigma \\
\delta a \\
\delta \phi
\end{array}\right)+\lambda\left(\begin{array}{cccc}
0 & 0 & 0 & -8 \\
0 & 0 & 0 & 4 D \\
0 & 0 & v^{\prime}+2 \sigma & -2 D^{2} \\
8 & 4 D & -2 D^{2} & 0
\end{array}\right)\left(\begin{array}{c}
\delta v \\
\delta \sigma \\
\delta a \\
\delta \phi
\end{array}\right)
\end{aligned}
$$

where:

$$
\begin{aligned}
& P_{v v}:=0, \quad P_{v \sigma}:=-4\left(1-v^{2}\right) \\
& P_{v a}:=-2 v v^{\prime}+2\left(1-v^{2}\right) D, \quad P_{v \phi}:=-16 a-4 \sigma D+4 v D^{2} \\
& P_{\sigma v}:=-P_{v \sigma}, \quad P_{\sigma \sigma}:=-4 v v^{\prime}+4\left(1-v^{2}\right) D \\
& P_{\sigma a}:=2 v \sigma^{\prime}+2 v v_{x}-2 \sigma v D+2 v^{2} D^{2}, \quad P_{\sigma \phi}:=8 \phi v+8 a D-4 v D^{3} \\
& P_{a v}:=-P_{v a}, \quad P_{a \sigma}:=-2 \sigma v^{\prime}+2 v v_{x}-2 \sigma v D+2 v^{2} D^{2}, \\
& P_{a a}:=-\phi+4 a \sigma+\phi v^{2}-2 v a^{\prime}+\sigma \sigma^{\prime}+2 a v^{\prime}-v \sigma_{x}+\sigma v_{x}-v v^{\prime \prime \prime}-v v_{x} D-v v^{\prime} D^{2}-v^{2} D^{3} \\
& P_{a \phi}:=-4 \phi \sigma-4 v \phi^{\prime}+2 \phi v D-4 a D^{2}-2 \sigma D^{3}+2 v D^{4} \\
& P_{\phi v}:=16 a+4 \sigma^{\prime}+4 v_{x}-4 \sigma D+4 v D^{2} \\
& P_{\phi \sigma}:=-8 \phi v+8 a^{\prime}+4 v^{\prime \prime \prime}+\left(8 a+4 v_{x}\right) D+4 v^{\prime} D^{2}+4 v D^{3} \\
& P_{\phi a}:=4 \phi \sigma+2 v \phi^{\prime}+2 \phi v^{\prime}-4 a_{x}-2 \sigma^{\prime \prime \prime}-2 v_{x x}+\left(2 \phi v+2 \sigma_{x}\right) D \\
&+\left(4 v_{x}-4 a-2 \sigma^{\prime}\right) D^{2}+2 \sigma D^{3}-2 v D^{4} \\
& P_{\phi \phi}:=-8 \phi_{x}-4 \phi^{\prime} D-12 \phi D^{2}+4 D^{5}
\end{aligned}
$$


It is found by inspection that the Poisson pencil of Table 2 can be restricted to the submanifold $v=\sigma=0$; the restricted biHamiltonian structure is again the one of the Laberge-Mathieu theory of Table 1. Clearly, this alternative way to obtain the $\mathrm{sKdV}$ of Laberge-Mathieu is less direct than the previous one, based on the choice (5.3), (5.4) for $A$ and $B$.

In conclusion, even though the biHamiltonian reduction of loop algebras can be performed in principle for alternative choices of $A$ and $B$, the choice corresponding to our adaptation of the Drinfeld-Sokolov criterion seems to give the SKdV theories in the more straightforward fashion. Of course, this remark does not exclude the possibility that different pairs $A$ and $B$ can be employed to obtain from loop superalgebras the biHamiltonian structures of different superintegrable systems. This possibility has a counterpart in the classical framework: for example, it is known [New] that the reduction of the loop algebra of $s l(2)$ gives the $\mathrm{KdV}$ theory if the Drinfeld-Sokolov criterion is applied for choosing the distinguished elements in the Lie algebra, whereas an alternative choice gives the AKNS theory.

\section{Concluding Remarks}

In the previous sections, our attention has been centered on the superextension of one relevant aspect of the Drinfeld-Sokolov theory; the other aspect of this theory, i.e. the relation between Lie superalgebras and the Lax formalism, has not been analyzed. We think it can be useful to conclude the paper with a few informal remarks on this subject, already considered in [IK].

From the geometrical viewpoint adopted in this paper, the Lax operator for a supersymmetric hierarchy is a supplementary output of the biHamiltonian reduction of loop superalgebras.

Indeed, assume we are given a Lie superalgebra $g$, the corresponding loop superalgebra and a biHamiltonian hierarchy on the quotient space $\mathscr{M}=\mathscr{S} / \mathscr{E}$. Let us pick up a section $m \mapsto S_{m}$ of the bundle $\mathscr{S} \stackrel{\pi}{\rightarrow} \mathscr{M}$, and realize the Lie superalgebra $g$ in terms of $(p+q) \times(p+q)$ matrices with entries in $\Lambda$. Viewing $S_{m}$ as a matrixvalued function on $K$, we define for each $m \in \mathscr{M}$ a matrix superdifferential operator

$$
\begin{gathered}
\mathscr{L}_{m}: \mathscr{F}\left(K, \Lambda^{p+q}\right) \rightarrow \mathscr{F}\left(K, \Lambda^{p+q}\right), \\
\Psi \mapsto \mathscr{L}_{m} \Psi:=D \Psi+\left(S_{m}+\lambda A\right) \Psi,
\end{gathered}
$$

depending on the parameter $\lambda$, and acting on column vectors formed by $p+q$ superfields; the matrix $A$ is as in (3.6). The Lax operator for the hierarchy can be obtained from the above matrix operator by means of a suitable procedure.

For example, in the case discussed in Sect. 4, Eqs. (4.4) and (4.16) imply that, at any point $m=\left(\begin{array}{c}a \\ \phi\end{array}\right)$ of $\mathscr{M}$ :

$$
\mathscr{L}_{m}\left(\begin{array}{c}
\psi \\
\psi_{1} \\
\psi_{2} \\
\psi_{3}
\end{array}\right)=D\left(\begin{array}{l}
\psi \\
\psi_{1} \\
\psi_{2} \\
\psi_{3}
\end{array}\right)+\left(\begin{array}{cccc}
0 & 0 & 1 & 0 \\
0 & 0 & 0 & 1 \\
0 & -1 & 0 & 0 \\
a+\lambda & 0 & \phi & 0
\end{array}\right)\left(\begin{array}{l}
\psi \\
\psi_{1} \\
\psi_{2} \\
\psi_{3}
\end{array}\right) .
$$

It is easily checked that the equation $\mathscr{L}_{m} \Psi=0$ implies $\psi_{1}=D^{2} \psi, \psi_{2}=-D \psi$, $\psi_{3}=-D^{3} \psi$ and

$$
\left(-D^{4}+\phi D-a\right) \psi=\lambda \psi
$$


Equation (6.3) is the eigenvalue problem for the well known Lax operator of the Manin-Radul theory.

By applying this technique to $g=\operatorname{osp}(2,2, \Lambda)$ we have obtained the Lax operator for the Laberge-Mathieu theory. The details of this construction will be reported in a forthcoming paper [MP], where the superalgebraic interpretation of the Lax formalism for the supersymmetric $\mathrm{KdV}$ theories will be systematically discussed.

Acknowledgements. We gratefully acknowledge P. Casati, L.A. Ibort, F. Magri and M. Pedroni for stimulating discussions. In particular, we are indebted to P. Casati for valuable support concerning Lie superalgebras. This paper has been partially supported by Consiglio Nazionale delle Ricerche, G.N.F.M., and by Ministero dell'Università e della Ricerca Scientifica, Progetto "Metodi Geometrici e Probabilistici in Teorie di Campo."

\section{References}

[Ber] Berezin, F.: Introduction to superanalysis. Dordrecht: D. Reidel 1987

[CMP] Casati, P., Magri, F., Pedroni, M.: BiHamiltonian manifolds and the $\tau$-function. Proceedings of the 1991 Joint Summer Research Conference on Mathematical aspects of Classical Field Theory (M. Gotai, J. Marsden, V. Moncrief, eds.), in Contemporary Math. 132, 213-234 (1992)

[Cor] Cornwell, J.: Group theory in Physics Vol. III. London: Academic Press 1989

[CP] Casati, P., Pedroni, M.: Drinfeld-Sokolov reduction on a simple Lie algebra from the biHamiltonian point of view. Lett. Math. Phys. 25, 89-101 (1992)

[DS] Drinfeld, V., Sokolov, V.: Lie algebras and equations of Korteweg-de Vries type. Sov. J. Mat. 30, 1975-2036 (1985)

[FMR] Figueroa-O'Farill, J., Mas, J., Ramos, E.: Integrability and biHamiltonian structure of the even order sKdV hierarchies. Rev. Mat. Phys. 3, 479-501 (1991)

[FSS] Frappat, L., Sciarrino, A., Sorba, P.: Structure of basic Lie superalgebras and of their affine extensions. Commun. Math. Phys. 121, 457-500 (1989)

[GO] Gursus, M., Oguz, O.: A super AKNS scheme. Phys. Lett. 108 A, 437-440 (1985)

[IK] Inami, T., Kanno, H.: Lie superalgebraic approach to super Toda lattice and generalized super KdV equations. Commun. Math. Phys. 136, 519-542 (1991)

[Ino] Inoue, A.: Foundation of real analysis on the superspace $\mathscr{R}^{m, n}$ over the $\infty$-dimensional Fréchet-Grassmann algebra. J. Fac. Sci. Univ. Tokio, Sect. IA Math. 39, 419-474 (1992)

[JP] Jadczyk, A., Pilch, K.: Superspaces and supersymmetries. Commun. Math. Phys. 78, 373-390 (1981)

[Kac] Kac, V.: Lie superalgebras. Advances in Math. 26, 8-96 (1977); Infinite-dimensional algebras, Dedekind's $\eta$-functions, classical Möbius function and the very strange formula. Advances in Math. 30, 85-136 (1978)

[Kup] Kupershmidt, B.: A super Kortwerg-de Vries equation. Phys. Lett. 102A, 213-215 (1984)

[Lei] Leites, D.: Introduction to the theory of supermanifolds. Russ. Math. Surv. 35, 1-64 (1980)

[LM] Laberge, C., Mathieu, P.: $N=2$ superconformal algebra and integrable $O(2)$ fermionic extension of the KdV equations. Phys. Lett. 215 B, 718-722 (1988)

[LMV] Landi, G., Marmo, G., Vilasi, G.: Remarks on the complete integrability of dynamical systems with fermionic variables. Quaderno 256, Dipartimento di Scienze Mathematiche, Univ. Trieste (1992)

[Mag] Magri, F.: A simple model of the integrable Hamiltonian equation. J. Math. Phys. 19, 1156-1162 (1978)

[MaR] Marsden, J.E., Ratiu, T.: Reduction of Poisson Manifolds. Lett. Math. Phys. 11, 161-169 (1986)

[Mat] Mathieu, P.: Supersymmetric extension of the Korteweg-de Vries equation. J. Math. Phys. 29, 2499-2506 (1988)

[MP] Morosi, C., Pizzocchero, L.: in preparation 
[MR] Manin, Y., Radul, A.: A supersymmetric extension of the Kadomtsev-Petviashvili hierarchy. Commun. Math. Phys. 98, 65-77 (1985)

[New] Newell, A.: Solitons in Mathematics and Physics. Philadelphia, PA: S.I.A.M. 1985

[Nij] Nijenhuis, A.: $X_{n-1}$-forming sets of eigenvectors. Proc. Kon. Ak. Ned. Wet. Amsterdam A 54, 200-212 (1951)

[OP] Oevel, W., Popowicz, Z.: The bi-Hamiltonian structure of fully supersymmetric Kortweg-de Vries systems. Commun. Math. Phys. 139, 441-460 (1991)

[Sem] Semenov-Tian Shansky, M.: What is a classical $r$-matrix?. Funct. Anal. Appl. 17, 259-272 (1983)

[SS] Salam, A., J., Strathdee, J.: Superfields and Fermi-Bose Symmetry. Phys. Rev. D 11, $1521-1535(1975)$

[VV] Vladimirov, V., Volovic, I.: Superanalysis I. Differential Calculus. Theor. Math. Phys. 59, 317-335 (1984); Superanalysis. II. Integral Calculus. Theor. Math. Phys. 60, 743-765 (1985)

Communicated by N.Yu. Reshetikhin 